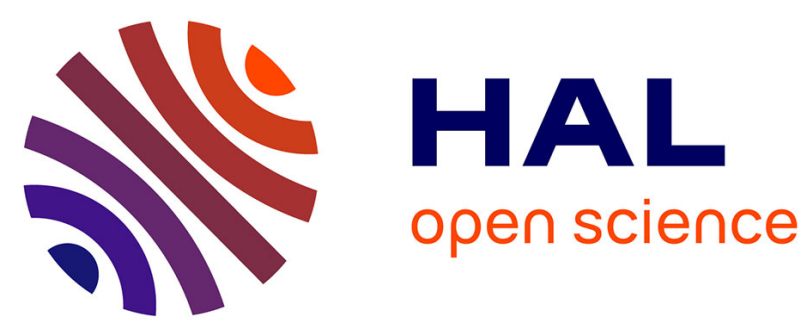

\title{
A New Methodology to Predict the Magnetic Shielding Effectiveness of Enclosures at Low Frequency in the Near Field
}

Amin Frikha, Mohamed Bensetti, Fabrice Duval, Nabil Benjelloun, Frédéric Lafon, Lionel Pichon

\section{To cite this version:}

Amin Frikha, Mohamed Bensetti, Fabrice Duval, Nabil Benjelloun, Frédéric Lafon, et al.. A New Methodology to Predict the Magnetic Shielding Effectiveness of Enclosures at Low Frequency in the Near Field. IEEE Transactions on Magnetics, 2015, 51 (3), pp.8000404. 10.1109/TMAG.2014.2362953 . hal-01235444

HAL Id: hal-01235444

https://hal-centralesupelec.archives-ouvertes.fr/hal-01235444

Submitted on 20 Aug 2020

HAL is a multi-disciplinary open access archive for the deposit and dissemination of scientific research documents, whether they are published or not. The documents may come from teaching and research institutions in France or abroad, or from public or private research centers.
L'archive ouverte pluridisciplinaire HAL, est destinée au dépôt et à la diffusion de documents scientifiques de niveau recherche, publiés ou non, émanant des établissements d'enseignement et de recherche français ou étrangers, des laboratoires publics ou privés. 


\title{
A New Methodology to Predict the Magnetic Shielding Effectiveness of Enclosures at Low Frequency in the Near Field
}

\author{
A. Frikha ${ }^{1}$, M. Bensetti ${ }^{2}$, F. Duval ${ }^{3}$, N. Benjelloun ${ }^{4}$, F. Lafon $^{5}$ and L. Pichon ${ }^{1}$ \\ ${ }^{1}$ LGEP, UMR 8507 CNRS, SUPELEC, Université Paris-Sud, Université Pierre et Marie Curie, \\ Plateau de Moulon, 11 rue Joliot Curie, 91192 Gif-sur-Yvette cedex, France. \\ ${ }^{2}$ SUPELEC, Département Energie, Plateau de Moulon, 3 rue Joliot Curie, 91192 Gif-sur-Yvette cedex, France. \\ ${ }^{3}$ CESI, 1 rue G. Marconi, Parc de la Vatine, 76137 Mt St Aignan Cedex, France. \\ ${ }^{4}$ ESIGELEC/IRSEEM, Avenue Galilée, 76801 Saint- Etienne- du-Rouvray, France. \\ ${ }^{5}$ VALEO, 2 Rue André Boulle, 94046 Créteil, France.
}

\begin{abstract}
In this paper, a methodology to calculate the shielding effectiveness (SE) of enclosures with slots at low frequency in the near magnetic field is presented. This modeling technique is based on the magnetic moments and the transmission line (TL) approach and makes specific assumptions. It is applied to a slot of small size relative to the dimensions of the enclosure. The slot is electrically short. This technique is validated in the case of a real enclosure with numerical simulations and experimental data. This method is dedicated to the case of mechatronics boxes using in electric and hybrid vehicles (EHVs), it allows obtaining the results with sufficient accuracy precision for dimensioning a magnetic shielding in a short time.
\end{abstract}

Index Terms—Enclosure, Low frequency, Magnetic moment, Magnetic field, Shielding effectiveness, Slot, Near field.

\section{INTRODUCTION}

$I^{N}$ N EHVs, high magnetic fields are generated due to high $\mathbf{1}$ electric currents. It may be necessary to control the level of electromagnetic field and assess the protection of electronic equipment located in the vehicle. Such protection can be provided by electromagnetic shielding, cabling architecture or additional equipment. The metallic enclosures are used in electronic devices to prevent electromagnetic interference (EMI). In these enclosures, it may be found apertures for ventilation or cabling purposes, slots and gaskets between different mechanical parts.

To calculate the shielding effectiveness, the numerical methods are an important area in electromagnetic compatibility (EMC) research [1]. Recently, many works have been developed about field coupling with metallic enclosure's aperture at high frequency [2]-[4]. In the case of EHV embedded systems, the far field conditions are not met. This may be due to a small distance from the source to the shield and/or the short distance between the shield and the measuring point. In this context, a method of characterization in the near field for the evaluation of the shielding effectiveness of the enclosure with slot is required. Therefore a modeling and a test bench are developed in this study.

The problem of predicting the shielding effectiveness of enclosures in the near field at low frequency is complex. This is due to several constraints such as diffusion, non-linear materials, multi-scale... The presence of slots in enclosures increases the difficulty of modeling; they make the numerical methods heavy with respect to the mesh and to the computation time and often need specific modeling techniques [3], [4]. In fact, the practical shielding effectiveness of enclosures is not determined only by material characteristics but also by

Manuscript received May 16, 2014. Corresponding author: A. Frikha (email: amin.frikha@lgep.supelec.fr).

Digital Object Identifier inserted by IEEE apertures in the shielding [2].

To overcome these constraints, a new solution is presented in this paper. It combines two modeling approaches. The first one devoted to the influence of slots is based on the magnetic moment approximation of a perfectly conducting infinite plane [2], [3], [5] where an adequate discretization is used according to the length of the slot. The second one uses an analytical method (TL approach) [6], [7] to take into account the diffusion phenomenon. The modeling process is validated in the near magnetic field at low frequency.

\section{SHIELDING EFFECTIVENESS OF AN INFINITE PLANE WITH A SLOT}

In [2], [3], [5] the aperture is replaced by magnetic and/or electric dipoles as shown in Fig.1. Usually in near magnetic field; the tangential component is not uniform in the slot. In this paper, we have investigated a new way to take into account this constraint by imposing an array of dipoles in the slot, this approach of modeling is strictly valid only for electrically small slot, i.e., the largest dimension is short compared to the wavelength.

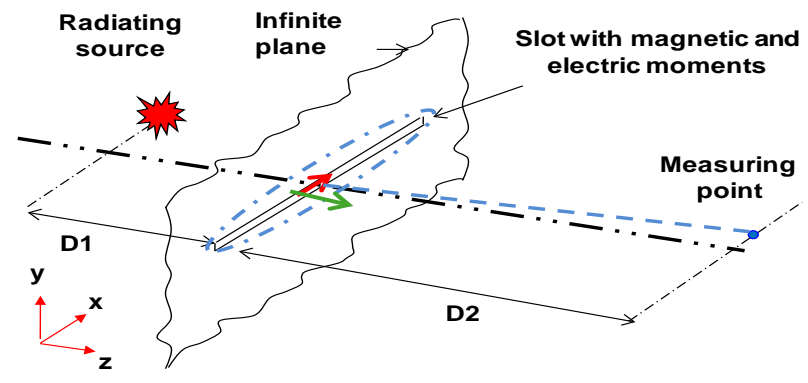

Fig. 1. Infinite plane with slot.

1) Magnetic Moment Approximation

The penetration of electromagnetic fields through an electrically small aperture could be calculated using the electric 
and magnetic equivalent dipoles as shown in Fig. 1 [2], [3], [5]. Neglecting the contribution of the electric dipole moment which is caused by the low value of electric polarizability, the electromagnetic properties of a slot can be described only by a magnetic dipole moment which is determined by equation (1) [2], [3].

$\vec{m}_{x}=-\alpha_{m, x x} \vec{H}_{x}^{s c}$

where $\alpha_{m, x x}$ is the longitudinal magnetic polarizability and $\vec{H}_{x}^{s c}$ is the short circuit magnetic field at the conducting surface in the absence of the aperture (equal to two times incident field $\left(H_{x}\right)$ ).

The magnetic polarizability depends only on the shape and size of the slot. Its values for different opening forms (circle, ellipse, rectangle or slot) can be calculated analytically, numerically or by measurement [5], [8]. In this study we have used an analytical method where the influence of the thickness of the slot $(1 \mathrm{~mm})$ on the value of the magnetic polarizability is taken into account following [8].

This paper will only consider two configurations for the radiating source: coplanar and coaxial. The coaxial configuration means that the axis of the radiating loop and that of the slot are parallel, otherwise the axis are perpendicular for the coplanar configuration.

In the first case (coplanar) the magnetic field is on the same axis $(+\hat{x})$ as the slot. The magnitude is non uniform on the slot's surface. So the slot is discretized with small elements where the magnetic field $\left(H_{x, i}\right)$ is supposed constant. In each element, the magnetic moments $\left(m_{x, i}\right)$ are affected and calculated using $\left(H_{x, i}, l, \alpha_{m, x x}\right)$ as shown in Fig. 2. The magnetic field radiated by the slot is calculated using the contribution of each magnetic moment with equation (2) and standard formulation of magnetic moment radiation [1] .

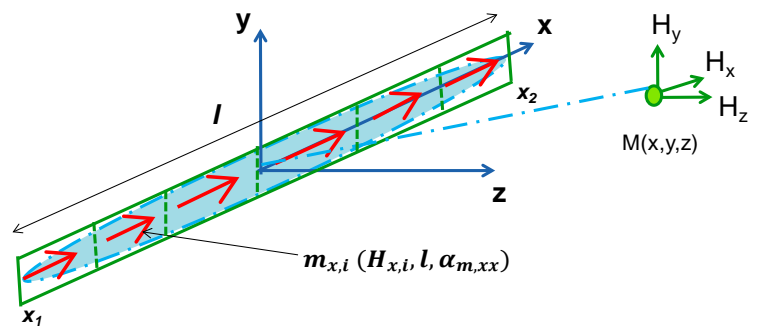

Fig. 2. Magnetic moment dipole distribution in slot (case 1).

$H^{x y z}=\frac{1}{\left|x_{2}-x_{1}\right|} \int_{x_{1}}^{x_{2}} H_{i}^{x y z} d x$

where $x_{2}, x_{1}$ are the limits of slot. $H_{i}^{x y z}$ is the magnetic field due to each discritization at $\mathrm{M}(\mathrm{x}, \mathrm{y}, \mathrm{z}) . H^{x y z}$ is the total magnetic field due to the slot at $\mathrm{M}(\mathrm{x}, \mathrm{y}, \mathrm{z})$.

In the second case (coaxial) the magnetic field is on the same axis with two orientations $(+\hat{x}$ and $-\hat{x})$. The magnitude is non uniform on the slot's surface. The slot is divided into two parts where the magnetic field has the same direction. For each part, the radiated magnetic field is calculated using a same procedure as in the previous case; the magnetic moments $\left(m_{x, i}\right)$ are calculated using $\left(H_{x, i}, l_{j}, \alpha_{m, x x, j}\right)$ instead of $\left(H_{x, i}, l, \alpha_{m, x x}\right)$ as shown in Fig. 3 and equation (3). The total radiated field of the slot is the sum of the radiated fields' vectors from each part.

The same process modeling is applied if the magnetic field in the slot changes its directions more than two times (general case of radiating source).

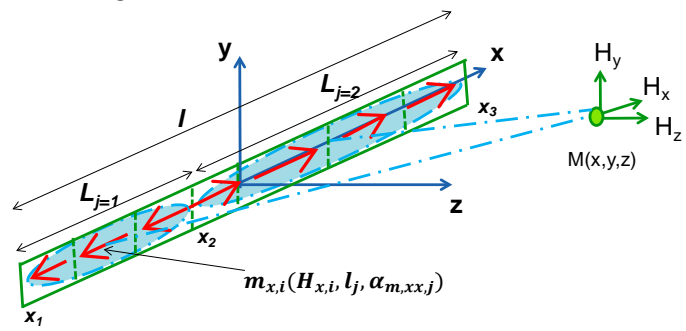

Fig. 3. Magnetic moment dipole distribution in slot (case 2).

$H^{x y z}=\frac{1}{\left|x_{2}-x_{1}\right|} \int_{x_{1}}^{x_{2}} H_{i}^{x y z} d x+\frac{1}{\left|x_{3}-x_{2}\right|} \int_{x_{2}}^{x_{3}} H_{i}^{x y z} d x$

where $x_{1}, x_{2}, x_{3}$ are the limits of each part.

2) Experimental Validation

To validate the method a test bench is developed as shown in Fig. 4. To simulate an infinite plane with a slot, a finite plane which has sufficiently large dimensions $\left(400 \times 400 \times 1 \mathrm{~mm}^{3}\right)$ is placed on a large cavity wall. The slot of $100 \mathrm{~mm}$ length and 1 $\mathrm{mm}$ width is located in the middle of a finite plane.

The developed test bench is constituted by a function generator which delivers a power of $24 \mathrm{dBm}$ to the developed radiating loop (8 turns and $23 \mathrm{~mm}$ diameter) on the whole frequency range. Using a receiver measuring, the induced voltage on a receiving loop (60 mm diameter, ROHDE \& SCHWARZ) is measured on the ( $9 \mathrm{kHz}-10 \mathrm{MHz})$ frequency range. The shielding effectiveness is obtained by finding the ratio of induced voltage without and with the shield as following [6]:

$S E=20 \log \left(\frac{V_{\text {without }}}{V_{\text {with }}}\right)$

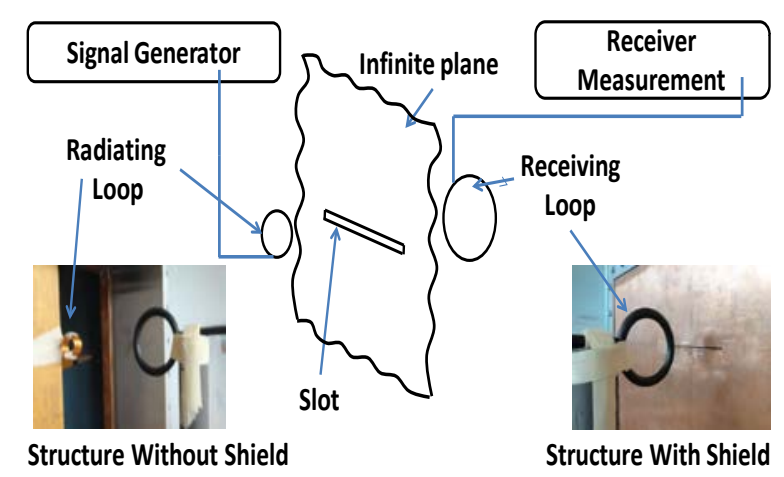

Fig. 4. Developed test bench.

It is important to account for the finite dimensions of the radiating loop. Thus, the distance between the radiating loop and the shield must be greater than the radiating loop diameter. It leads to assume that the radiated field produced by the radiating loop is the same radiated field produced by the magnetic dipole. In the interested frequency range, the source 
with this dimension will be small compared to the radiated wavelength and can be approximated as an elementary dipole [9].

In the developed models, the shielding effectiveness is calculated using the magnetic field in the center of the receiving loop. The results can be improved by calculating the induced voltage on the receiving loop using the magnetic field distribution over the surface described by the antenna [6].

3) Analysis of Results

In order to validate the model, different cases have been studied. The effect of the radiating loop position (coaxial, coplanar) and the distance between the radiating loop and the shield $(50 \mathrm{~mm}, 70 \mathrm{~mm}$ ) are presented in Fig. 5. A good agreement is obtained between the simulated results (developed model, CST micro wave) and the measured data. The difference at low frequency $(9 \mathrm{kHz})$ which can reach $5 \mathrm{~dB}$ between the simulated results and the measured can be explained as follows. In the modeling process, the infinite plane has a perfect electrical conductivity, it means that no diffusion through the walls, however in the experiments, a copper material of $1 \mathrm{~mm}$ of thickness is used. The induced voltage is due to the radiated field from the slot and the diffusion through the walls (especially for coaxial configuration). In addition, in the modeling process, the effect of the reflected fields on the radiating loop is not taken into account. However, this phenomenon degrades the radiation properties of the radiating loop by Lenz's law during measurements.
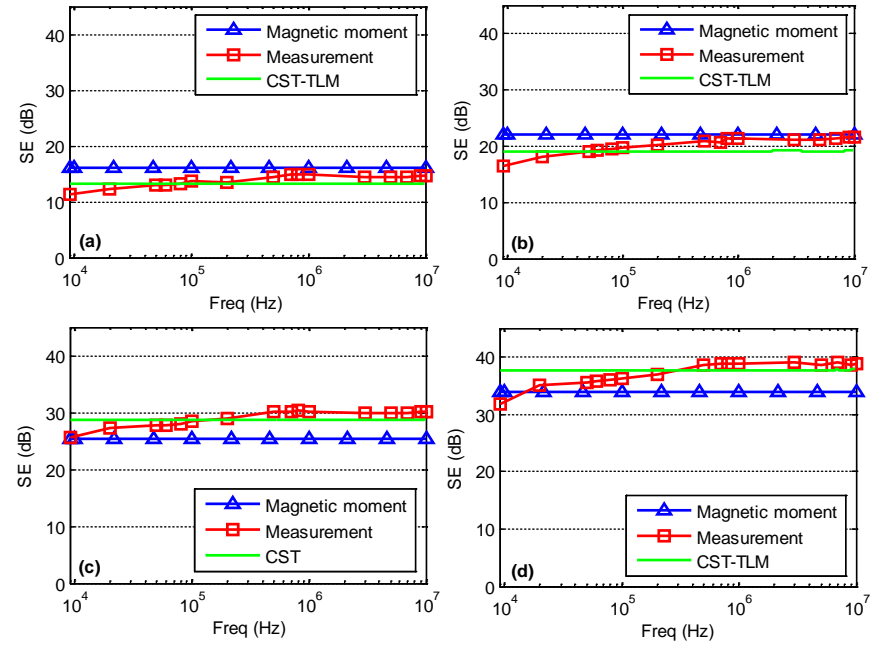

Fig. 5. Shielding effectiveness of infinite plane. Coplanar configuration: (a) $50 \mathrm{~mm}$, (b) $70 \mathrm{~mm}$. Coaxial configuration: (c) $50 \mathrm{~mm}$, (d) $70 \mathrm{~mm}$.

The results show that the shielding effectiveness increases with the distance between the source and the shield distance. Moreover it takes for same distance higher value for the coaxial configuration than coplanar configuration thus a good configuration to shield.

In this study, the numerical modeling requests $13 \mathrm{Mb}$ of memory and 5 hours of computational time. However, the method based on magnetic moment needs only $2 \mathrm{Mb}$ and 2 seconds.
III. MAGNETIC SHIELDING EFFECTIVENESS OF AN ENCLOSURE WITH A SLOT

\section{A. Methodology}

In order to calculate the shielding effectiveness in the case of an enclosure with a slot, we have combined the shielding effectiveness of an infinite plane with and without slot as show in Fig. 6. In the developed methodology the magnetic dipole approximation is used to calculate the shielding effectiveness of an infinite perfectly conducting plane with a slot which has a perfect electrical conductivity. However, the TL approach is used to calculate the shielding effectiveness of an infinite plane without slot which has real physical properties.

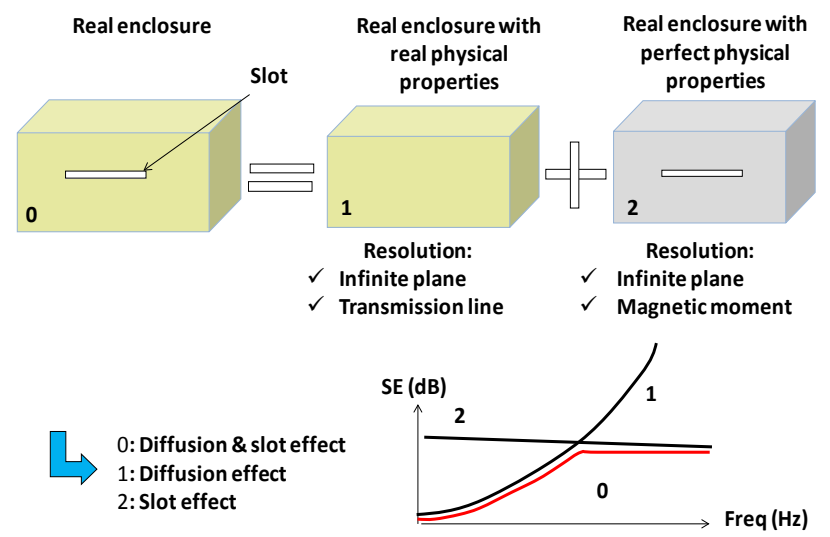

Fig. 6. Shielding of enclosures using proposed methodology

An infinite plane of electrical conductivity, electrical permittivity, magnetic permeability and finite thickness is considered. The shield is excited by the far field source. The shielding effectiveness can be calculated by the TL approach [6], [7].

As known, the shielding effectiveness depends on the type of source (plane wave, near electric field, near magnetic field). In this study, we will focus on near magnetic field. The following results are obtained using a simple approximation by replacing the intrinsic impedance of free space $\left(\eta_{0}=377 \Omega\right)$, with the wave impedance for the small magnetic dipole $Z_{m}$ [7].

Concerning the shielding effectiveness of an infinite plane with slot that has a perfect electrical conductivity, we apply the developed method which based on magnetic dipole approximation described in the previous section.

\section{B. Experimental Validation}

We have applied the methodology in the case of a simple enclosure made on copper; the enclosure has $500 \times 400 \times 200$ $\mathrm{mm}^{3}$ dimension and $1 \mathrm{~mm}$ thickness. A slot of $100 \mathrm{~mm}$ length and $1 \mathrm{~mm}$ width is located in the middle of the enclosure surface which has $400 \times 200 \mathrm{~mm}^{2}$ dimensions. The enclosure is excited by a radiating loop at $50 \mathrm{~mm}$. The magnetic field is measured inside the enclosure at $50 \mathrm{~mm}$ far from slot. In this part we have interested only by coplanar configuration which leads to the worst shielding effectiveness.

To validate the methodology, we have developed a test bench like the first one shown before in section II. An enclosure with a slot is implemented instead of an infinite plane (Fig. 7). The measurements are performed outside the anechoic chamber in 
the presence and absence of the enclosure.
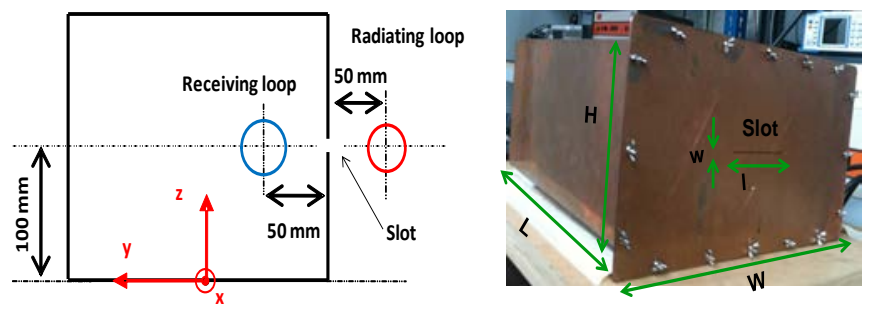

Fig. 7. Modeled structure

Fig. 8 shows the results which obtained by the developed model, a TL approach, CST microwave studio and a measurement. The results of different models have a good agreement with accuracy of $2 \mathrm{~dB}$ in high frequencies. It is the same at low frequencies where the TL approach has been validated. However, in the middle frequency range, the penetration of magnetic field is due by both the diffusion and the slot thus the little bit difference between models. In addition, we will accept some errors for using the TL approach which mainly due to small dipole approximation.

The shielding effectiveness which is weak at low frequency increases and reaches high values at the onset of the skin effect. Whereas, the shielding effectiveness of the slot remains almost constant. However, the shielding effectiveness of an enclosure with a slot follows the shielding effectiveness of the simple infinite plane up to the intersection frequency and then switches to follow the effectiveness of the slot. It is clear that the measuring in the frequency range $(9 \mathrm{kHz}-10 \mathrm{MHz})$, the penetration of the magnetic field through a walls (diffusion) is neglected compared by the penetration through the slot.

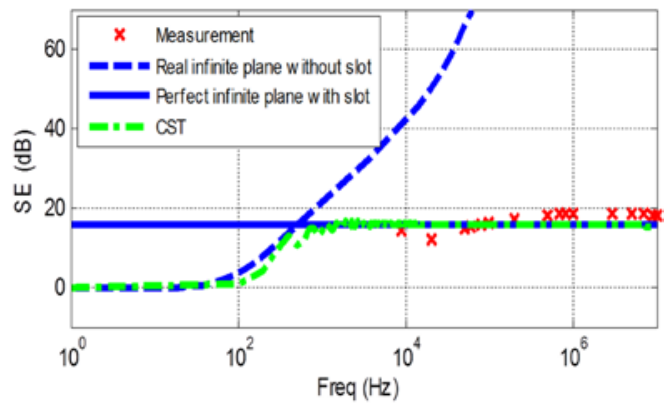

Fig. 8. The shielding effectiveness of enclosure

In this study, the numerical simulations required about $20 \mathrm{Mb}$ and about 12.5 hours to achieve the solution using Intel (R) Xeon (R), CPU E31225 @ 3.10 GHz, 3.10 GHz, and 8 Go of memory. However, the proposed methodology needs only $4 \mathrm{Mb}$ of memory and 5 seconds of computational time.

\section{Limit of the Methodology}

We keep the same dimensions of the enclosure and we change the length of the slot from $60 \mathrm{~mm}$ to $200 \mathrm{~mm}$. The goal is to see the limits of the methodology to calculate the shielding effectiveness of real application. The obtained results by numerical and analytical simulations at $10 \mathrm{MHz}$ are presented in Fig. 9. The methodology is limited by the ratio between enclosure and slot dimensions. In our study, we find a difference of $2.2 \mathrm{~dB}$ if the ration equal to 2.85 .

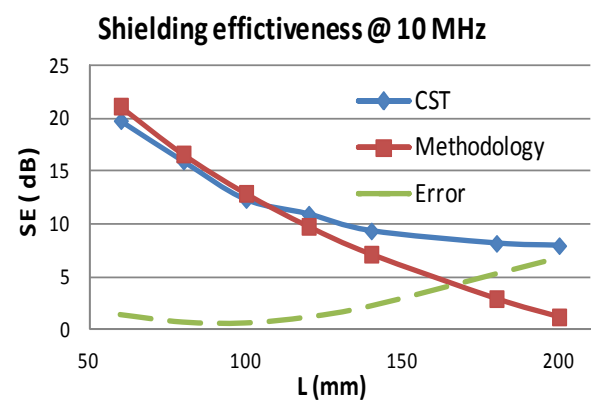

Fig. 9. Limit of methodology at $10 \mathrm{MHz}$

\section{CONCLUSION}

Through this work, we have presented a new method based on magnetic moment approximations to calculate the shielding effectiveness of an infinite plane with a slot in the near magnetic field at low frequency. To avoid the constraints of numerical techniques, the slot is treated as an array of magnetic moment dipoles. This method is combined with a TL approach to estimate the shielding effectiveness of an enclosure with a slot taking into account the diffusion through the walls.

Even if the results are obtained with a somewhat crude approximation, the methodology allows finding the shielding effectiveness in short time. The methodology is limited by the ratio between the enclosure and slot dimensions. On the next step, we will adapt this methodology for the applications with slot which have arbitrary dimensions.

\section{REFERENCES}

[1] S. Celozzi, R. Araneo, and G. Lovat, Electrompagnetic Shielding. Hoboken, NJ: Wiley-IEEE, 2008.

[2] D. Pouhè, and G. Monich, "Assessments of Shielding Effectiveness of Gaskets by Means of the Modified Bethe's Coupling Theory, " IEEE Trans. Electromagn. Compat., vol. 50, no. 2, pp. 305-315, May. 2008.

[3] T. Martin, M. Backstrom, and J. Lorén, "Semi-Empirical Modeling of Apertures for Shielding Effectiveness Simulations," IEEE Trans. Electromagn. Compat., vol. 45, no. 2, pp. 229-237, May. 2003.

[4] R. Araneo, and G. Lovat, "Fast MoM Analysis of the Shielding Effectiveness of Rectangular Enclosures With Apertures, Metal, Plates, and Conducting Objects,' IEEE Trans. Electromagn. Compat., vol. 51, no. 2, pp. 274-283, May. 2009.

[5] F. De Meulenaere, and J. Van Bladel, "Polarizability of Some Small Apertures,” IEEE Trans. Antennas Propag., vol. AP-25, no. 2, pp. 198205, Mar. 1977.

[6] R. Araneo, and S. Celozzi, "Exact Solution of the Low-Frequency Coplanar Loops Shielding Configuration,” IEE Proc. Sci., Meas. Tech., vol. 149, no. 1, pp. 37-44, Jan. 2002.

[7] C. R. Paul, Introduction to Electromagnetic Compatibility. 2nd ed. Hoboken, N. J. Chichester: Wiley Interscience; John Wiley, 2006.

[8] L. K. Warne, T. E. Koontz, and K. C. Chen, Equivalent Polarizabilities of Apertures with Depth. AFWL Interaction Note 474, pp. 1-99, Mar. 1989.

[9] J.L. Cocquerelle, and C. Pasquier, Rayonnement Electromagnétique des Convertisseurs à Découpage: Approche Simplifiée par le Concept des Ilots Rayonnants. EDP Sciences, 2002. 